\title{
Perspectives on the mesenchymal origin of metastatic cancer
}

\author{
Leanne C. Huysentruyt • Thomas N. Seyfried
}

Published online: 14 September 2010

(C) The Author(s) 2010. This article is published with open access at Springerlink.com

\begin{abstract}
Emerging evidence suggests that many metastatic cancers arise from cells of the myeloid/macrophage lineage regardless of the primary tissue of origin. A myeloid origin of metastatic cancer stands apart from origins involving clonal evolution or epithelial-mesenchymal transitions. Evidence is reviewed demonstrating that numerous human cancers express multiple properties of macrophages including phagocytosis, fusogenicity, and gene/protein expression. It is unlikely that the macrophage properties expressed in metastatic cancers arise from sporadic random mutations in epithelial cells, but rather from damage to an already existing mesenchymal cell, e.g., a myeloid/macrophage-type cell. Such cells would naturally embody the capacity to express the multiple behaviors of metastatic cells. The view of metastasis as a myeloid/ macrophage disease will impact future cancer research and anti-metastatic therapies.
\end{abstract}

Keywords Metastatic cancer - Myeloid/macrophage lineage $\cdot$ Epithelial-mesenchymal transitions $\cdot$ Phagocytosis

\section{Introduction}

Cancer is a complex disease resulting in tumors composed of multiple cell types with diverse biological characteristics $[1,2]$. Cells within the primary tumor can differ with respect to origin, growth rate, karyotype, drug sensitivity,

\section{T. N. Seyfried $(\bowtie)$}

Biology Department, Boston College,

Chestnut Hill, MA, USA

e-mail: Thomas.seyfried@bc.edu

\section{C. Huysentruyt}

Department of Medicine, Hematology and Oncology,

University of California, San Francisco,

San Francisco, CA, USA

e-mail: leanne.huysentruyt@ucsf.edu and metastatic potential [2-4]. While each cell type within the primary tumor contributes to the malignancy of the disease, cells that acquire the abilities to invade and to metastasize are responsible for the majority of cancer related deaths $[1,5]$. Despite the clinical importance of metastasis, much remains unknown about the origin and development of this disease.

Metastasis involves the spread of cancer cells from the primary tumor to surrounding tissues and distant organs. The metastatic cascade is a series of sequential and interrelated steps to include cancer cell detachment from the primary tumor, intravasation into the circulation, evasion of immune attack, extravasation at a distant capillary bed, and invasion and proliferation in distant organs [6-10]. Metastatic cells also establish a microenvironment through the release of cytokines and growth factors that facilitate angiogenesis and proliferation, resulting in macroscopic, malignant secondary tumors. In addition, metastatic cells preferentially invade those organs (lymph nodes, lung, liver, brain, bone, pleura, and peritoneum) that promote tumor cell growth and survival consistent with the "seed and soil" hypothesis [8, 11-14]. While the major steps of metastasis are well documented, the process by which a metastatic cell arises from a population of non-metastatic cells in a primary tumor is largely unknown [15-17].

Several mechanisms have been advanced to account for the origin of metastasis. It is widely thought that metastatic cancer cells arise from pre-existing tumor cells that have undergone additional genetic alterations during the later stages of tumor progression, a process known as the clonal origin of metastasis $[1,9,18-20]$. The epithelial-mesenchymal transition (EMT) suggests that metastatic cells arise through a step-wise accumulation of gene mutations that eventually transform an epithelial cell into a tumor cell with mesenchymal features $[1,9,18-21]$. The idea for an EMT origin comes from findings that many cancers generally 
arise in epithelial tissues where abnormalities in cell-cell and cell-matrix interactions occur during tumor progression [22]. Eventually, neoplastic cells emerge that appear as mesenchymal cells, which lack cell-cell adhesion and are dysmorphic in shape [17]. These transformed epithelial cells eventually acquire the multiple effector mechanisms of metastasis [17]. Recent studies suggest that ectopic coexpression of only two genes might be all that is necessary to facilitate EMT in some gliomas [23]. Considerable controversy surrounds the EMT hypothesis of metastasis, as evidence of EMT is not often detected in tumor pathological preparations [22, 24, 25].

Based on numerous similarities between macrophages and metastatic cancer cells, we propose that many metastatic cancers arise from cells of the myeloid lineage. More specifically, metastatic cells arise from resident or infiltrating macrophages or myeloid cells of the tumor stroma that become neoplastic during disease progression. As mesenchymal cells [26, 27], myeloid/macrophage-type cells would naturally embody to the capacity to express multiple behaviors of metastasis. The basis for this hypothesis comes from our recent findings from unique metastatic tumors that arose spontaneously in the inbred VM mouse strain [14, 28]. Two of these highly metastatic/invasive tumors express multiple properties of macrophages. It is unlikely that the metastatic properties of these mouse tumors are unique, as a review of the literature indicates that these mouse tumors share multiple properties with most types of human metastatic cancers. The similarities between the mouse and human metastatic cancers raise the possibility that metastasis is a disease of myeloid cells regardless of tissue origin. Our goal is to highlight the similarities of myeloid cells (especially macrophages) and metastatic cancer cells, and to discuss the possible mechanisms by which these cells could become neoplastic.

\section{Linkage of macrophages with metastatic cancer}

Macrophages have long been considered the origin of human metastatic cancers; however, this has not been widely accepted or recognized [12, 29-32]. Rather than being considered part of the neoplastic cell population, macrophages are generally considered part of the tumor stroma as macrophages form a large portion of the inflammatory cell infiltrate in most cancer types [33, 34]. Depending on the tumor type, tumor-associated macrophages (TAMs) can constitute up to $80 \%$ of the total tumor mass [35]. TAMs are known to facilitate tumor development and progression by establishing a pre-metastatic niche and by enhancing tumor inflammation and angiogenesis [33, 36-39].

Macrophages are among the most versatile cells of the body with respect to their ability to migrate, to change shape, and to secrete growth factors and cytokines [14, 4042]. Macrophages are known to have two distinct polarization states: the classically activated (or M1) and the alternatively activated (or M2). Macrophages acquire the M1 phenotype in response to pro-inflammatory molecules and release inflammatory cytokines, reactive oxygen species, and nitric oxide [34, 43-46]. In contrast, macrophages acquire the M2 phenotype in response to antiinflammatory molecules such as IL-4, IL-13, IL-10, and apoptotic cells [43, 47]. Additionally, M2 macrophages are poor antigen presenters, immunosuppressive, and promote tissue remodeling and repair [34]. Emerging evidence suggests that M1 and M2 macrophages have distinct roles during tumor initiation and malignant progression [48].

M1 macrophages are believed to facilitate the early stages of tumorigenesis through the creation of an inflammatory microenvironment that produces DNA and mitochondrial damage [48, 49]. However, after the establishment of the primary tumor, during tumor progression, TAMs generally undergo a phenotypic switch and acquire the M2 polarization state [43]. The M2 TAMs scavenge debris and promote tumor growth, angiogenesis, and metastasis [34, 43, 44].

Increasing evidence suggests that myeloid/macrophage cells are also part of the malignant cell population. Aichel first proposed over a century ago that tumor progression involved fusion between leukocytes and somatic cells (reviewed in [31]). Several human metastatic cancers are known to express multiple molecular and behavioral characteristics of macrophages to include phagocytosis, cell-cell fusion, and antigen expression (Table 1). We suggest that an origin from myeloid cells can account for many properties of metastatic cancer.

\section{The wound-healing M2 macrophage and the metastatic cell: behavioral similarities}

Interestingly, macrophages express most hallmarks of metastatic tumor cells when responding to tissue injury or disease. For example, monocytes extravasate from the vasculature and are recruited to the wound via cytokines released from the damaged tissue. Within the wound, monocytes differentiate into alternatively activated macrophages and dendritic cells where they release a variety of pro-angiogenic molecules including vascular endothelial growth factor, fibroblast growth factor, and platelet-derived growth factor [126, 127]. M2 macrophages also actively phagocytose dead cells and cellular debris [42, 128]. On occasion, macrophages undergo homotypic fusion resulting in multinucleated giant cells with increased phagocytic capacity [29, 129]. Following these wound-healing activities, macrophages intravasate back into the circulation where they travel to the lymph nodes to participate in the 
Table 1 Tumors expressing macrophage characteristics

\begin{tabular}{llll}
\hline Tumor & Phagocytosis & Fusogenicity & Gene expression \\
\hline Bladder & {$[50]$} & & \\
Brain & {$[14,51-54]$} & {$[55]$} & {$[14,54]$} \\
Breast & {$[56-63]$} & {$[64-68]$} & {$[69-71]$} \\
Carcinoma of unknown primary & {$[72]$} & {$[73]$} & \\
Endometrial & {$[74]$} & & \\
Fibrosarcoma & {$[63]$} & & {$[81-84]$} \\
Gall bladder & & {$[75]$} & \\
Liver & & {$[76]$} & {$[96,99-101]$} \\
Lung & {$[57,77-80]$} & {$[67]$} & {$[102]$} \\
Lymphoma/leukemia & {$[85-87]$} & {$[88-91]$} & \\
Melanoma/skin & {$[92-96]$} & {$[32,97,98]$} & {$[106]$} \\
Meth A sarcoma & {$[102]$} & {$[102]$} & {$[108]$} \\
Multiple myeloma & {$[103]$} & {$[104]$} & {$[110]$} \\
Ovarian & {$[63,105]$} & & {$[111]$} \\
Pancreatic & {$[107,108]$} & {$[109]$} & \\
Rectal & & {$[112,113]$} & \\
Renal & {$[111]$} & {$[12,15,29-31,89,120-125]$} & \\
Rhabdomyosarcoma & {$[114,115]$} & & \\
Reviews & {$[116-119]$} & & \\
\hline
\end{tabular}

immune response [42, 130, 131]. These findings indicate that normal macrophages are capable of intravasation, tissue invasion, release of pro-angiogenic molecules/cytokines, survival in hypoxic and necrotic environments, and extravasation, i.e., hallmark behaviors of metastatic tumor cells.

\section{Phagocytosis: a shared behavior of M2 macrophages and metastatic cells}

Phagocytosis, the engulfment and ingestion of extracellular material, is a specialized behavior of M2 macrophages and other professional phagocytes [42]. This process is essential for maintaining tissue homeostasis by clearing apoptotic cells, cellular debris, and invading pathogens [42]. Interestingly, many malignant tumor cells are phagocytic both in vitro and in vivo (Table 1). Macrophages also express high levels of lysosomal-enriched cathepsins, which facilitate the digestion of proteins ingested following phagocytosis or pinocytosis [132, 133]. This is interesting since lysosomal cathepsins D and B are viewed as prognostic factors in cancer patients [133]. Indeed, a high content of these enzymes in tumors of the head and neck, breast, brain, colon, or endometrium was considered a sign for high malignancy, high metastasis, and overall poor prognosis [133].

The phagocytic behavior of tumor cells was first described over a century ago from histopathological observations of foreign cell bodies within in the cytoplasm of cancer cells, which displayed crescent-shaped nuclei [134]. This cellular phenotype, commonly referred to as either "birds-eye" or "signet-ring", is the result of the ingested material pushing the nucleus to the periphery of the phagocytic cell [116]. While this phagocytic/cannibalistic phenomenon is commonly seen in feeding microorganisms, cell cannibalism is an exclusive property of malignant tumor cells in humans [116]. These tumor cell phagocytic/cannibalistic behaviors are not to be confused with autophagy, a cellular self-digestion process often associated with starvation conditions [135, 136]. It has been reported that both human and murine cancers can phagocytose other tumor cells, erythrocytes, leukocytes, platelets, dead cells, as well as extracellular particles (Table 1) [56, 77, 116].

\subsection{Phagocytic cancers}

Numerous reports have described the phagocytic behaviors seen in aggressive human cancers and in some murine tumors (Table 1). We previously identified two spontaneous invasive/metastatic murine brain tumors (VM-M2 and VMM3) that express many macrophage behaviors including phagocytosis [14]. While extracranial metastasis of central nervous system tumors is not common, many gliomas, especially glioblastoma multiforme, are highly metastatic if the tumor cells can gain access to extraneural sites [14, 137-141]. Moreover, extracranial metastasis portends an 
extremely poor survival, with the vast majority of patients surviving less than 6 months from the diagnosis of metastatic disease [142].

The phagocytic activity of the metastatic VM-M2 and VM-M3 tumor cells was similar to that of the RAW 264.7 macrophage cell line [14]. Similar findings were reported for the methylcholanthrene-induced murine P388 mouse lymphoma cells, which display macrophage morphology, form rosettes, phagocytose latex beads, and strongly adhere to glass and plastic surfaces [85]. These findings indicate that some mouse tumor cell lines can manifest the phagocytic behavior seen in macrophages and in numerous human metastatic cancers.

While phagocytic behaviors have been reported for most forms of human cancer including skin, breast, lymphoma, lung, brain, ovarian, pancreatic, renal, endometrial, rhabdomyosarcoma, myeloma, fibrosarcoma, and bladder, not all cancer cells within a tumor are phagocytes (Table 1). For most of the tumors described, phagocytosis was restricted primarily to those cells that are highly invasive and metastatic [14, 51, 52, 56-58, 78, 79, 92, 93, 107]. Lugini et al. measured the phagocytic behavior of cell lines derived from primary human melanomas $(n=8)$ and metastatic lesions $(n=11)$ [92]. Interestingly, the phagocytic behavior all of the cell lines derived from metastatic lesions was similar to that of the macrophage controls, whereas phagocytic behavior was not found in any of the cell lines derived from primary melanomas [92]. Histological examination of in vivo metastatic melanoma lesions confirmed the presence of phagocytic tumor cells [93]. Similar findings of phagocytosis were reported for human metastatic breast cancer [56]. Numerous phagocytic tumor cells were identified within metastatic breast cancer lesions and were not observed within the primary tumor of the same patient [56]. Additionally, breast cancer malignancy and grade correlates with the number of phagocytic tumor cells present within the tumor stroma [59].

\subsection{Targeting phagocytosis}

Several investigators suggested that tumor cell phagocytosis could be targeted as a potential therapy for metastatic cancers. For example, Ghoneum et al. showed that MCF-7 breast cancer cells undergo apoptosis after engulfing yeast cells either in vitro or in vivo [58,60]. Phagocytosis of yeast cells also effectively induces apoptosis in human cancers of the gastrointestinal tract including tongue, squamous cell carcinoma, and colon adenocarcinoma [143]. These reports suggest that the phagocytic behavior of metastatic tumor cells can be targeted for the development of new anti-metastasis therapies.

Additionally, the phagocytic activity of metastatic melanoma cells is significantly increased when the cells are grown under low glucose conditions suggesting that metastatic cells use phagocytosis as a way to "feed" when nutrient supplies are low [93, 94]. Therefore, a metastasis targeted therapy could be effective if administered to energy-stressed metastatic tumor cells. Dietary energy restriction (DR) is an effective means to reduce circulating glucose levels and induce energy stress in tumor cells. DR can also reduce inflammation and tumor angiogenesis while increasing tumor cell apoptosis [144-148]. Moreover, DR increases macrophage phagocytosis [149]. Hence, DR administered in combination with an anti-phagocytosis targeted therapy could potentially reduce primary tumor size, vascularity, and the number of metastatic tumor cells.

\subsection{Phagocytosis for diagnostics}

Effective resection of invasive/metastatic tumors can be improved if the margins between tumor tissue and normal tissue are readily identified. In order to identify rat C6 glioma cells that invaded beyond the main tumor mass, Zimmer et al. used monocrystalline iron oxide nanoparticles that the tumor cells could phagocytose as a contrasting agent [150]. This was able to identify gliomas cells that had invaded into the rat parenchyma. Viewed collectively, these studies suggest that the phagocytic behavior of tumor cells can be exploited for therapeutic strategies and development of new diagnostic/imaging protocols.

\section{Fusogenicity}

Fusogenicity is the ability of a cell to fuse with another cell through the merging of their plasma membranes [129]. This process can be easily induced in vitro as is seen with the formation of antibody-producing hybridomas. However, cell fusion in humans is a highly regulated complex process that is essential for fertilization (sperm and egg), and skeletal muscle (myoblasts) and placenta (trophoblast) formation. Outside of these developmental processes, cellto-cell fusion is normally restricted to differentiated cells of myeloid origin (reviewed in [120]). During differentiation, subsets of macrophages fuse with each other to form multinucleated osteoclasts in bone or multinucleated giant cells in response to foreign bodies [29]. Osteoclasts and giant cells have increased cell volume that facilitates engulfment of large extracellular materials [29]. Macrophages are also thought to fuse with damaged somatic cells during the process of tissue repair $[29,129,151,152]$.

In addition to homotypic fusion, macrophages are known to undergo heterotypic fusion with tumor cells $[29,30,120$, 153]. Aichel first suggested in 1911 that fusion between somatic cells and leukocytes could induce aneuploidy 
resulting in tumors with increased malignancy (reviewed in [32]). Nearly 60 years later, Mekler and Warner proposed that fusion of committed tumor cells with host myeloid cells would produce tumor hybrids capable of migrating throughout the body and invading distant organs [121, 154]. Recently, this hypothesis has received more attention with the findings reported by John Pawelek and colleagues $[30,31,69,97,98,112,113,155]$. They also suggested that these hybrids could account for the diversity of cell phenotypes within tumors [121, 154]. Fusion between tumor cells and myeloid cells, with subsequent nuclear fusion, could produce new phenotypes in the absence of new mutations, as the hybrids would express genetic and functional traits of both parental cells [32]. These neoplastic hybrids would have the ability of macrophages to intravasate, to extravasate, and to migrate to distant organs while also possessing the unlimited proliferative potential of the cancer cells. Since myeloid cells are part of the immune system, tumor hybrids would also be able to evade immune surveillance [25].

\subsection{Fusogenic cancers}

Fusogenic tumor cells are found in a wide variety of cancer types including, melanoma, breast, renal, liver, gall bladder, lymphoma, and brain (Table 1). Tumor cell hybrids can form either in vitro or in vivo from fusions between two tumor cells or between a tumor cell and a normal somatic cell. One of the first reports of tumor cell fusion hybrids showed that human glioma cells, when implanted within the cheeks of hamsters, spontaneously fused with nontumorigenic host cells, resulting in metastatic hybrid human-hamster tumor cells [55]. Many of the early reports for fusogenic cancers described fusions between lymphomas and myeloid cells. For example, spontaneous in vivo fusion between the non-metastatic murine MDW4 lymphoma and host bone marrow cells resulted in aneuploid metastatic tumor cells [88].

Munzarova et al. recognized that numerous traits expressed in macrophages were also expressed in metastatic melanoma cells and suggested that the tumor metastasis could result from fusions between tumor cells and macrophages $[12,122]$. Rachkovsky et al. tested this hypothesis by inducing fusions between cultured non-metastatic Cloudman S91 melanoma cells and murine peritoneal macrophages. The majority of the resulting macrophagemelanoma hybrids displayed increased metastatic potential when grown in vivo [32]. Further studies revealed that the Cloudman S91 melanoma cells could undergo spontaneous fusion with the murine host cells in vivo resulting in secondary lesions that were comprised mostly of tumorhost cell hybrids. The authors concluded that the tumor cells likely fused with host myeloid cells [97]. Artificial fusions of human monocytes and mouse melanoma cells revealed that the resulting hybrids expressed both human and mouse genes [98]. Other investigators also showed that the macrophage-specific antigens F4/80 and Mac-1 were expressed in murine Meth A sarcoma cells after spontaneous in vivo fusion with host cells. Interestingly, latex bead phagocytosis was also expressed in the Meth A sarcoma-host cell fusion hybrids [102]. Since these fusion hybrids expressed genotypes and phenotypes of both parental cells, it appears that the non-metastatic tumor cells could acquire an invasive/metastatic phenotype without new mutations.

Fusion among tumor cells in human solid tumors is difficult to detect. Recent reports, however, have provided direct evidence for fusions between tumor cells and myeloid cells in human bone marrow transplant (BMT) recipients $[112,113]$. Both radiation therapy and immunosuppression can increase the incidence of metastatic cancers [156]. DNA analysis of micro-dissected metastatic cells from a child diagnosed with renal cell carcinoma after a BMT revealed DNA from both the BMT donor and the recipient in the metastatic cells [112]. Bone marrow and tumor cell hybrids were also identified in a female who developed renal carcinoma after receiving a BMT from a male donor [113]. These reports provided the first genetic evidence that spontaneous fusions can occur between human myeloid cells and tumor cells.

It is well documented that tumor-associated macrophages promote tumor progression in many cancers through the release of cytokines, and pro-angiogenic and prometastatic molecules (reviewed in [33, 38]). However, the fusion of cancer cells with tissue macrophages could also accelerate tumor progression. The tumor hybrid daughter cells could acquire the migratory/invasive behavior from the macrophage genome while still maintaining unlimited proliferative potential. Macrophage-macrophage fusions could also induce aneuploidy resulting in a tumorigenic myeloid cell $[30,123]$. The significance of macrophage fusion hybrids in human metastatic cancer requires more attention. The numerous in vitro studies and in vivo reports suggest that myeloid hybrids are responsible for the metastatic progression of at least a subset of cancers. Multinucleated giant cells, a signature of hybrid formation, are frequently seen in human cancers, suggesting that cell fusions are not rare events (Table 1). Regardless of the mechanism, metastatic cells express numerous behaviors of mesenchymal/myeloid cells and, if exploited, could generate novel therapeutic strategies for managing metastatic cancers.

\section{Tumor cell expression of myeloid antigens}

Myeloid cells express a wide variety of markers that are unique to their ontogeny and function [157]. Routine 
histological and immunohistochemical analyses are often preformed to assess tumor type and grade. Since TAMs are often correlated with a poor patient prognosis, tumor biopsies are frequently evaluated for macrophage markers. The macrophage antigen-expressing cells within the tumor stroma are usually classified as TAMs. However, several reports show that macrophage-specific antigens are expressed on a wide variety of human cancer cells (Table 1).

Ruff and Pert demonstrated that several macrophage antigens (CD26, C3bi, and CD11b) were expressed on tumor cells from small cell lung carcinoma (SCLC) [81]. Levels of expression were comparable to that seen in the monocyte controls. It is important to note that the macrophage antigens were expressed in the cultured tumor cells themselves and further confirmed in vivo. This eliminated the possibility that the antigen expression was derived from TAMs. These investigators concluded that the SCLC tumor cells in their specimens were not of lung origin, but rather were of myeloid origin. A malignant transformation of recruited myeloid cells, from smokingrelated tissue damage, was offered as an explanation for the origin of tumor cells with myeloid/macrophage properties [81]. Although this interpretation was controversial [70, 82], the authors demonstrated additional myeloid properties of these tumor cells [83]. Other investigators confirmed macrophage antigen expression on other SCLC tumors and cell lines [82, 84]. Myeloid-associated antigens (CD14 and $\mathrm{CD} 11 \mathrm{~b}$ ) were also expressed in five metastatic breast cancer cell lines [70]. None of the breast cancer cell lines, however, expressed markers for $\mathrm{B}$ or $\mathrm{T}$ cells [70]. The authors suggested that common antigen sharing between different cell types could be related to common cellular interactions [70]. In light of these findings and those from Ruff and Pert, we suggest that the shared antigen expression of macrophages and metastatic tumor cells is the result of a common mesenchyme origin.

Further evidence for a mesenchymal origin of metastatic cancer comes from tissue microarray analysis of 127 breast cancer patients [71]. CD163 was expressed on the tumor cells of $48 \%$ of the patients, while MAC387 was expressed on the tumor cells of $14 \%$ of the patients [71]. Pathology confirmed that the staining was localized to the tumor cells and not solely to the tumor infiltrating macrophages. Interestingly, cancers that contained CD163-expressing tumor cells had a more advanced histological grade, a higher occurrence of distant metastasis, and reduced patient survival [71]. This report demonstrated, for the first time, that tumor cells expressing macrophage antigens could be identified in more than half of breast cancer patients. Similar studies were conducted on 163 patients with rectal cancer [110]. CD163 was expressed in $31 \%$ of the rectal tumors from patients in the preoperative irradiation group, but in only $17 \%$ in the non-irradiation group. Prognosis was also worse for those patients with CD163-positive cancer cells than in those patients with CD163-negative cancer cells [110]. These findings are consistent with role of radiation in inducing tumor cell-macrophage fusions and in exacerbating the metastatic properties of some cancers [71, $110,158]$. These studies demonstrated that macrophage antigens, which are associated with enhanced metastasis and poor prognosis, are expressed on the tumor cells of patients with breast and rectal cancers.

\section{Carcinoma of unknown primary origin}

Carcinoma of unknown primary (CUP) is a systemic metastatic disease without an identifiable primary tumor and is often associated with poor prognosis. Approximately $5 \%$ of all newly diagnosed cancers are classified as CUP $[159,160]$. Histologically, these cancers are usually classified as adenocarcinomas, squamous cell carcinomas, poorly differentiated carcinoma, and neuro-endocrine carcinomas [160]. It is thought that these aggressive cancers rapidly metastasize before the primary tumor has had time to develop into a macroscopic lesion [160]. Interestingly, aneuploidy was identified in 70\% of CUP adenocarcinoma, suggesting that these cancers contain hybrid cells [29, 73]. Reports have also demonstrated that CUP contain signet-ring cells, indicating that a subset of these cancers exhibit phagocytic behavior [72]. Due to the highly aggressive nature of these tumors, we suggest that some CUPs could have a myeloid/macrophage origin.

\section{Many cancers express multiple macrophage properties}

The evidence presented in this review indicates that many metastatic cancers can express multiple myeloid characteristics (Table 1). For instance, many tumors that were phagocytic or fusogenic also expressed myeloid antigens, further supporting a myeloid origin of these metastatic cancers. It is important to note that the myeloid properties we highlighted were expressed in the tumor cells themselves and should not be confused with myeloid properties expressed by tumor-associated macrophages, which are also present in the tumors but are not tumorigenic.

\section{Possible mechanisms}

Emerging evidence indicates that cancer is a metabolic disease, regardless of tissue or cellular origin, which arises as a result of impaired cellular energy metabolism and 
mitochondrial dysfunction (reviewed by [22]). Numerous studies indicate that tumor mitochondria are abnormal and are incapable of generating normal levels of energy [49, 161-166]. Defective mitochondria often arise as the result of damage to mitochondrial membrane lipids, specifically cardiolipin, through mutagens/carcinogens, radiation, hypoxia, inflammation, reactive oxygen species, and inherited mutations that alter mitochondrial energy production [49]. It is currently believed that any impairment in mitochondrial energy formation can lead to genetic instability via the retrograde (RTG) response, a signaling pathway that consists of sensors such as HIF- $1 \alpha$ and NfkB that detect mitochondrial stability [167]. Activation of the RTG pathway results in the synthesis of ATP through glycolysis when respiratory function is impaired. Over time, this respiration impairment would result in the upregulation of the TCA cycle and glycolytic substrate level phosphorylation, and induce proliferation and genetic defects, resulting in malignant transformation [22, 167-169]. These findings indicate that the integrity of the nuclear genome is dependent upon the functionality of the mitrochondria for all cell types, including cells of myeloid and macrophage origin [22].

Metastatic myeloid tumor cells could arise from resident tissue macrophages or TAM that have suffered mitochondria damage (Fig. 1). The majority of tissues contain macrophages as part of their normal cellular composition and TAM are also a major cell type in most cancers where they can facilitate tumor progression $[33,42,157,158]$. In fact, depending on the tumor type, as noted above, up to $80 \%$ of the cells within a tumor are macrophages [33, 35]. Macrophages generally hone to mitochondria-damaging environments in response to inflammation, infection, would repair, and tumorigenesis [37, $38,126,128,170]$. It seems likely that mitochondria damage would occur resulting in cellular transformation through the RTG response where glucose and glutamine become the primary metabolic fuels for growth and survival $[158,171]$. Glucose and glutamine are also major energy metabolites for normal cells of myeloid/macrophage lineage [158, 172]. Damage to myeloid/macrophage respiratory function could lead to a reliance on substrate level phosphorylation for energy, resulting in a malignant macrophage with the highest metastatic potential [22].

Mitochondria are dynamic organelles that undergo regular fusion and fissions [173]; thus, abnormalities in mitochondrial lipid composition would be rapidly disseminated throughout the cell's mitochondrial network and could also be passed along to other cells through cytoplasmic inheritance during cellular fusion events. While cell fusion events are considered rare outside of normal developmental processes, various reports have demonstrated that the frequency of cell fusion can be up to $1 \%$ in in vivo tumor models [64, 120, 123]. Additionally, chronic inflammation increases fusion events [174, 175]. Considering the large number of cells within a solid tumor, the fusogenic cell population could comprise a significant portion of the tumor.

Fig. 1 Proposed mechanisms of macrophage transformation. The tumor microenvironment consists of numerous mitochondria-damaging elements which would likely result in impaired mitochondria energy production in TAM and tissue macrophages and subsequent genetic instability through the RTG response $(A)$. Macrophage fusion hybrids could result in cells able to express both the tumor and macrophage genomes resulting in cells with metastatic potential $(B)$

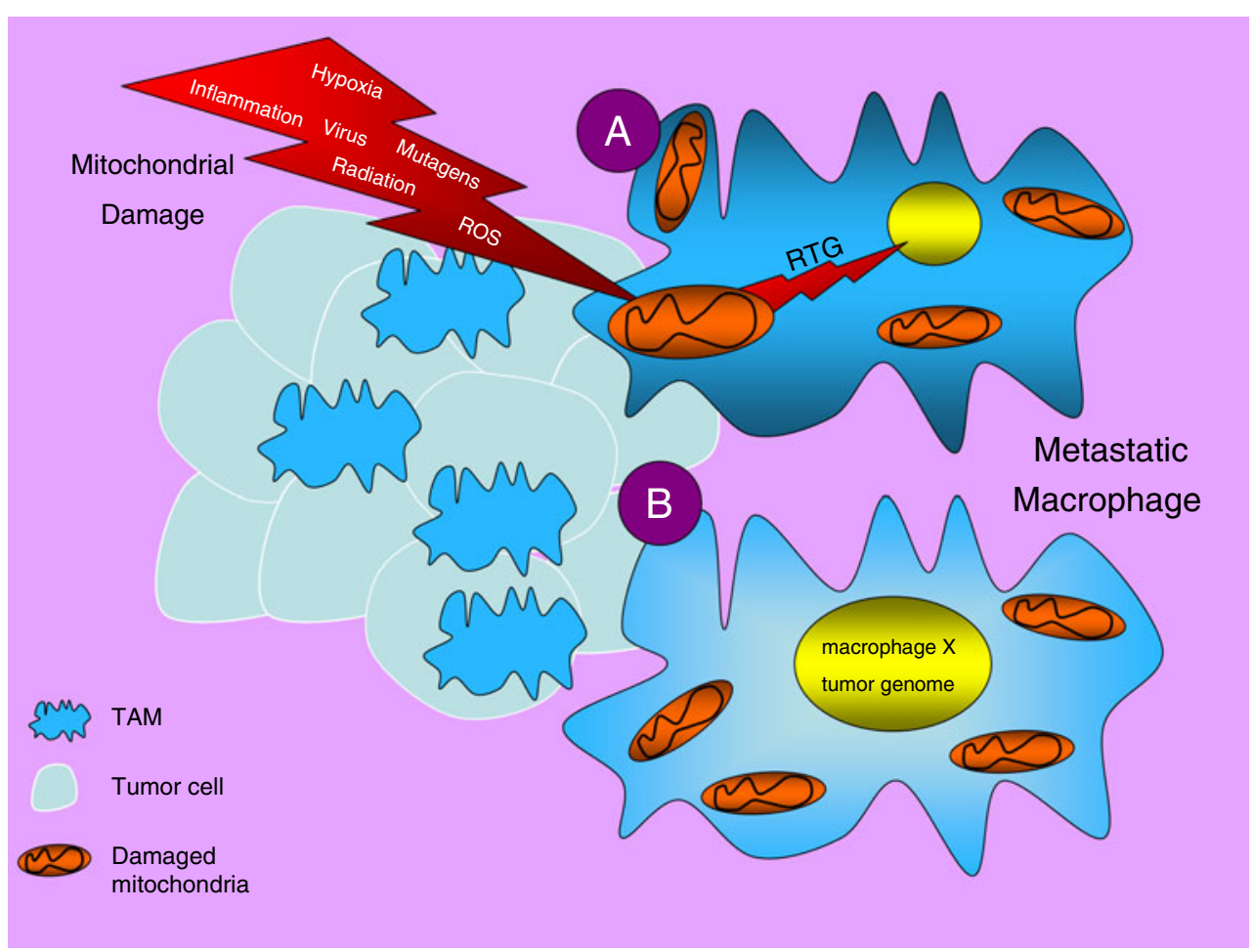


Cell fusion results in the mixing of the two parental cytoplasms [120, 129, 176]. Therefore, the formation of cancer cell and macrophage fusion hybrids would result in the transfer of damaged mitochondria from the cancer cell to the macrophage hybrid daughter cells. The subsequent inheritance of abnormal mitochondria would likely result in the transformation of the hybrid genome through the RTG response [22, 167-169]. In fact, the presence of fusion hybrids has been shown to correlate with tumor malignancy [65].

While it is unknown what triggers formation of macrophage $\times$ tumor hybrids, various steps in the macrophage response to tumor development could provide the opportunity for cell fusion. Within hypoxic tumor areas, macrophages digest apoptotic cells. It has been suggested that macrophages may abort cellular digestion resulting in hybrid formation [31]. Additionally, macrophages could also fuse with somatic cells during tissue repair. Since tumors represent unhealed wounds [33, 177], it is possible that macrophages could fuse with tumor cells in an attempt to "heal" the tissue. Radiation damage during therapeutic intervention will also enhance fusion leading to more aggressive and difficult to manage tumors [110, 158]. Fusion events could also arise through the action of tumorassociated viruses, as several different tumor types (leukemia, lymphoma, Kaposi sarcoma, hepatocellular carcinoma, anogenital cancers, etc.) are associated with viral infection $[124,153]$. Viral proteins enable successful cellular infection by fusing biological membranes. Additionally, some viruses utilize cellular fusion as a way to facilitate their spread (reviewed in [124]). Interestingly, several tumor-associated viruses including Epstein-Barr virus, Kaposi's sarcomaassociated herpes virus, human papilloma virus (HPV), hepatitis $\mathrm{B}$ and $\mathrm{C}$ viruses, Rous sarcoma virus, and human $\mathrm{T}$ cell leukemia virus type 1 localize to the mitochondria compartment of the cell where they could potentially cause mitochondria defects [178-181].

The macrophage fusion hypothesis is an attractive explanation for metastasis. Fusions among macrophages and tumor cells could also account for histological structures in metastatic sites that resemble the histology of the primary tumor tissue as it has been shown, through reprogramming strategies, that macrophages are capable of producing fully functional epithelial cells at secondary sites while retaining histological characteristics of the original primary tissue [123, 182, 183]. Tumor fusions could also explain aneuploidy and chromosomal abnormalities seen in most cancer cells $[31,124]$. However, there are conflicting observations showing suppressed tumorigenicity following hybridization between normal cells and tumor cells [22, 184].

Damage to the respiratory capacity of resident tissue phagocytes, TAM, or macrophage hybrids would trigger a RTG response and over time lead to uncontrolled proliferation and genomic instability. Metastatic behavior would be the expected outcome of impaired mitochondrial function in myeloid or macrophage cells, as these cell types are mesenchymal cells that are able to degrade the extracellular matrix, migrate through local tissues, and enter and exit the circulation.

\section{Modeling the myeloid origin of metastasis}

The development of more effective therapies for the management of human metastatic cancers can be improved with animal models that accurately reflect the human disease. We recently described two new mouse models of systemic metastatic cancer in the inbred VM mouse strain that express multiple myeloid/macrophages characteristics to include phagocytosis, morphological appearance, and gene and lipid expression [14, 28, 185]. The VM-M2 and the VM-M3 tumors model all major steps of metastasis including, local invasion, intravasation, immune system survival, extravasation, and secondary tumor formation [14, 28, 185]. Moreover, the VM-M2 and the VM-M3 tumor cells are naturally metastatic from any inoculated tissue and, in contrast to most other mouse metastatic models, do not require intravenous injections to initiate the metastatic phenotype. Tumor cells that possess true or natural metastatic potential should not require intravenous injection to demonstrate metastasis. We also showed that the response of the metastatic VM tumors to well described anti-cancer drugs (cisplatin and methotrexate) was similar to that described previously in human cancer patients treated with these drugs [28]. We suggest that these mouse models of systemic metastatic cancer will be useful for developing novel therapies that target the myeloid properties of metastasis.

\section{Concluding remarks}

A transition from an epithelial-type cell to a mesenchymaltype cell is often considered an underlying characteristic of metastasis. As an alternative to a series of gain-of-function mutation events, we suggest that the metastatic mesenchymal phenotype can arise from malignant myeloid cells either through cell hybrid formation or through direct transformation of tissue macrophages. We think that it is improbable that random mutations acquired through a Darwinian selection process could account for all of the myeloid-cell behaviors necessary for the completion of the metastatic cascade. It is in our opinion that the myeloid origin of metastasis is the most probable explanation of tumor progression to date. 
We suggest that future research in this field should focus on the myeloid properties of metastatic cells. The VM mouse model, as well as many of the human and murine cell lines discussed above, will be valuable for the development of novel metastasis therapies. Additionally, we urge others to consider the possibility that some of the cells previously identified as TAM in tumor pathological tissue specimens may in fact be metastatic tumor cells. This is especially true since TAMs are often localized to the leading edge of an invading tumor mass, and high levels of TAM are usually indicative of poor prognosis. We contend that targeting macrophage behaviors (e.g., phagocytosis, fusogenicity, and energy metabolism) would result in therapies that are effective in managing metastatic cancer, regardless of primary tumor origin.

Acknowledgements This work was supported in part by NIH grants (HD39722, NS055195, and CA102135), a grant from the American Institute of Cancer Research, and the Boston College Expense Fund. The authors would like to thank Michael S. McGrath and Laura M. Shelton for critical comments.

Authors' contribution Leanne C. Huysentruyt contributed to the conception, design, and writing of this manuscript. Thomas $\mathrm{N}$. Seyfried contributed to the conception, design, and editing of this manuscript. Both authors read and approved the final manuscript.

Open Access This article is distributed under the terms of the Creative Commons Attribution Noncommercial License which permits any noncommercial use, distribution, and reproduction in any medium, provided the original author(s) and source are credited.

\section{References}

1. Hanahan, D., \& Weinberg, R. A. (2000). The hallmarks of cancer. Cell, 100(1), 57-70.

2. Fidler, I. J., Kim, S. J., \& Langley, R. R. (2007). The role of the organ microenvironment in the biology and therapy of cancer metastasis. Journal of Cellular Biochemistry, 101(4), 927-936.

3. Ohgaki, H., \& Kleihues, P. (2009). Genetic alterations and signaling pathways in the evolution of gliomas. Cancer Science, 100(12), 2235-2241.

4. Wu, J. M., et al. (2008). Heterogeneity of breast cancer metastases: comparison of therapeutic target expression and promoter methylation between primary tumors and their multifocal metastases. Clinical Cancer Research, 14(7), 1938-1946.

5. Jemal, A., et al. (2007). Cancer statistics. CA: A Cancer Journal for Clinicians, 57(1), 43-66.

6. Welch, D. R. (2006). Defining a cancer metastasis. AACR education book 2006 (pp. 111-115). Philadelphia: American Association for Cancer Research.

7. Chambers, A. F., Groom, A. C., \& MacDonald, I. C. (2002). Dissemination and growth of cancer cells in metastatic sites. Nature Reviews Cancer, 2(8), 563-572.

8. Fidler, I. J. (2003). The pathogenesis of cancer metastasis: The 'seed and soil' hypothesis revisited. Nature Reviews Cancer, 3 (6), 453-458.
9. Duffy, M. J., McGowan, P. M., \& Gallagher, W. M. (2008). Cancer invasion and metastasis: Changing views. The Journal of Pathology, 214(3), 283-293.

10. Steeg, P. S. (2006). Tumor metastasis: Mechanistic insights and clinical challenges. Natural Medicines, 12(8), 895-904.

11. Joyce, J. A., \& Pollard, J. W. (2009). Microenvironmental regulation of metastasis. Nature Reviews Cancer, 9(4), 239-252.

12. Munzarova, M., \& Kovarik, J. (1987). Is cancer a macrophagemediated autoaggressive disease? Lancet, 1(8539), 952-954.

13. Paget, S. (1889). The distribution of secondary growths in cancer of the breast. Lancet, 1, 571-573.

14. Huysentruyt, L. C., et al. (2008). Metastatic cancer cells with macrophage properties: Evidence from a new murine tumor model. International Journal of Cancer, 123(1), 73-84.

15. Pawelek, J. M. (2008). Cancer-cell fusion with migratory bonemarrow-derived cells as an explanation for metastasis: New therapeutic paradigms. Future Oncology, 4(4), 449-452.

16. Steeg, P. S. (2008). Heterogeneity of drug target expression among metastatic lesions: Lessons from a breast cancer autopsy program. Clinical Cancer Research, 14(12), 3643-3645.

17. Bacac, M., \& Stamenkovic, I. (2008). Metastatic cancer cell. Annual Review of Pathology, 3, 221-247.

18. Fearon, E. R., \& Vogelstein, B. (1990). A genetic model for colorectal tumorigenesis. Cell, 61(5), 759-767.

19. Nowell, P. C. (2002). Tumor progression: A brief historical perspective. Seminars in Cancer Biology, 12(4), 261-266.

20. Nowell, P. C. (1976). The clonal evolution of tumor cell populations. Science, 194(4260), 23-28.

21. Kalluri, R., \& Weinberg, R. A. (2009). The basics of epithelialmesenchymal transition. The Journal of Clinical Investigation, $119(6), 1420-1428$.

22. Seyfried, T. N., \& Shelton, L. M. (2010). Cancer as a metabolic disease. Nutrition \& Metabolism, 7, 7.

23. Carro, M. S., et al. (2010). The transcriptional network for mesenchymal transformation of brain tumours. Nature, 463 (7279), 318-325.

24. Hart, I. R. (2009). New evidence for tumour embolism as a mode of metastasis. The Journal of Pathology, 219(3), 275-276.

25. Garber, K. (2008). Epithelial-to-mesenchymal transition is important to metastasis, but questions remain. Journal of the National Cancer Institute, 100(4), 232-3-239.

26. Banaei-Bouchareb, L., et al. (2006). A transient microenvironment loaded mainly with macrophages in the early developing human pancreas. The Journal of Endocrinology, 188(3), 467-480.

27. Mallat, M., Marin-Teva, J. L., \& Cheret, C. (2005). Phagocytosis in the developing CNS: More than clearing the corpses. Current Opinion in Neurobiology, 15(1), 101-107.

28. Huysentruyt, L. C., Shelton, L. M., \& Seyfried, T. N. (2009). Influence of methotrexate and cisplatin on tumor progression and survival in the VM mouse model of systemic metastatic cancer. International Journal of Cancer, 126, 65-72.

29. Vignery, A. (2005). Macrophage fusion: Are somatic and cancer cells possible partners? Trends in Cell Biology, 15(4), 188-193.

30. Pawelek, J. M., \& Chakraborty, A. K. (2008). Fusion of tumour cells with bone marrow-derived cells: A unifying explanation for metastasis. Nature Reviews Cancer, 8(5), 377-386.

31. Pawelek, J. M. (2000). Tumour cell hybridization and metastasis revisited. Melanoma Research, 10(6), 507-514.

32. Rachkovsky, M., et al. (1998). Melanoma $\times$ macrophage hybrids with enhanced metastatic potential. Clinical \& Experimental Metastasis, 16(4), 299-312.

33. Seyfried, T. N. (2001). Perspectives on brain tumor formation involving macrophages, glia, and neural stem cells. Perspectives in Biology and Medicine, 44(2), 263-282. 
34. Mantovani, A., et al. (2002). Macrophage polarization: Tumorassociated macrophages as a paradigm for polarized M2 mononuclear phagocytes. Trends in Immunology, 23(11), 549-555.

35. Morantz, R. A., et al. (1979). Macrophages in experimental and human brain tumors. Part 1: Studies of the macrophage content of experimental rat brain tumors of varying immunogenicity. Journal of Neurosurgery, 50(3), 298-304.

36. Talmadge, J. E., Donkor, M., \& Scholar, E. (2007). Inflammatory cell infiltration of tumors: Jekyll or Hyde. Cancer Metastasis Reviews, 26, 373-400.

37. Bingle, L., Brown, N. J., \& Lewis, C. E. (2002). The role of tumourassociated macrophages in tumour progression: Implications for new anticancer therapies. The Journal of Pathology, 196(3), 254-265.

38. Lewis, C. E., \& Pollard, J. W. (2006). Distinct role of macrophages in different tumor microenvironments. Cancer Research, 66(2), 605-612.

39. Pollard, J. W. (2008). Macrophages define the invasive microenvironment in breast cancer. Journal of Leukocyte Biology, 84 (3), 623-630.

40. Stossel, T. (1999). Mechanical responsesof white blood cells. In J. Snyderman (Ed.), Inflammation: Basic principles and clinical correlates (pp. 661-679). New York: Lippincott Williams \& Wilkins.

41. Gordon, S. (1999). Development and distribution of mononuclear phagocytes: Relevance to inflammation. In J. Gallin \& R. Snyderman (Eds.), Inflammation: Basic principles and clinical correlates (pp. 35-48). New York: Lippincott Williams \& Wilkins.

42. Burke, B., \& Lewis, C. E. (Eds.). (2002). The macrophage (2nd ed.). Oxford University Press: New York.

43. Biswas, S. K., Sica, A., \& Lewis, C. E. (2008). Plasticity of macrophage function during tumor progression: Regulation by distinct molecular mechanisms. Journal of Immunology, 180(4), 2011-2017.

44. Mantovani, A., \& Sica, A. (2010). Macrophages, innate immunity and cancer: Balance, tolerance, and diversity. Curr Opin Immunol, 22(2), 231-237.

45. Sica, A., Saccani, A., \& Mantovani, A. (2002). Tumor-associated macrophages: A molecular perspective. International Immunopharmacology, 2(8), 1045-1054.

46. Sica, A., et al. (2006). Tumour-associated macrophages are a distinct M2 polarised population promoting tumour progression: Potential targets of anti-cancer therapy. European Journal of Cancer, 42(6), 717-727.

47. Gordon, S. (2003). Alternative activation of macrophages. Nature Reviews. Immunology, 3(1), 23-35.

48. Qian, B. Z., \& Pollard, J. W. (2010). Macrophage diversity enhances tumor progression and metastasis. Cell, 141(1), 3951.

49. Kiebish, M. A., et al. (2008). Cardiolipin and electron transport chain abnormalities in mouse brain tumor mitochondria: Lipidomic evidence supporting the Warburg theory of cancer. Journal of Lipid Research, 49(12), 2545-2556.

50. Kojima, S., et al. (1998). Clinical significance of "cannibalism" in urinary cytology of bladder cancer. Acta Cytologica, 42(6), $1365-1369$.

51. Youness, E., et al. (1980). Tumor cell phagocytosis. Its occurrence in a patient with medulloblastoma. Archives of Pathology \& Laboratory Medicine, 104(12), 651-653.

52. Bjerknes, R., Bjerkvig, R., \& Laerum, O. D. (1987). Phagocytic capacity of normal and malignant rat glial cells in culture. Journal of the National Cancer Institute, 78(2), 279-288.

53. Kumar, P. V., Hosseinzadeh, M., \& Bedayat, G. R. (2001). Cytologic findings of medulloblastoma in crush smears. Acta Cytologica, 45(4), 542-546.
54. Leenstra, S., et al. (1995). Human malignant astrocytes express macrophage phenotype. Journal of Neuroimmunology, 56(1), 17-25. issn: 0165-5728.

55. Goldenberg, D. M., Pavia, R. A., \& Tsao, M. C. (1974). In vivo hybridisation of human tumour and normal hamster cells. Nature, 250(5468), 649-651.

56. Marin-Padilla, M. (1977). Erythrophagocytosis by epithelial cells of a breast carcinoma. Cancer, 39(3), 1085-1089.

57. Spivak, J. L. (1973). Phagocytic tumour cells. Scandinavian Journal of Haematology, 11(3), 253-256.

58. Ghoneum, M., \& Gollapudi, S. (2004). Phagocytosis of Candida albicans by metastatic and non metastatic human breast cancer cell lines in vitro. Cancer Detection and Prevention, 28(1), 17-26.

59. Abodief, W. T., Dey, P., \& Al-Hattab, O. (2006). Cell cannibalism in ductal carcinoma of breast. Cytopathology, 17 (5), 304-305.

60. Ghoneum, M., et al. (2007). Yeast therapy for the treatment of breast cancer: A nude mice model study. In Vivo, 21(2), 251258.

61. Ghoneum, M., et al. (2008). S. cerevisiae induces apoptosis in human metastatic breast cancer cells by altering intracellular $\mathrm{Ca} 2$ + and the ratio of Bax and Bcl-2. International Journal of Oncology, 33(3), 533-539.

62. Coopman, P. J., et al. (1998). Phagocytosis of cross-linked gelatin matrix by human breast carcinoma cells correlates with their invasive capacity. Clinical Cancer Research, 4(2), 507515 .

63. Lee, H., et al. (2007). Phagocytosis of collagen by fibroblasts and invasive cancer cells is mediated by MT1-MMP. Biochemical Society Transactions, 35(Pt 4), 704-706.

64. Lu, X., \& Kang, Y. (2009). Efficient acquisition of dual metastasis organotropism to bone and lung through stable spontaneous fusion between MDA-MB-231 variants. Proceedings of the National Academy of Sciences of the United States of America, 106(23), 9385-9390.

65. Miller, F. R., et al. (1988). Spontaneous fusion between metastatic mammary tumor subpopulations. Journal of Cellular Biochemistry, 36(2), 129-136.

66. Bjerregaard, B., et al. (2006). Syncytin is involved in breast cancer-endothelial cell fusions. Cellular and Molecular Life Sciences, 63(16), 1906-1911.

67. Mortensen, K., et al. (2004). Spontaneous fusion between cancer cells and endothelial cells. Cellular and Molecular Life Sciences, 61(16), 2125-2131.

68. Athanasou, N. A., et al. (1989). The origin and nature of stromal osteoclast-like multinucleated giant cells in breast carcinoma: Implications for tumour osteolysis and macrophage biology. British Journal of Cancer, 59(4), 491-498.

69. Handerson, T., et al. (2005). Beta1,6-branched oligosaccharides are increased in lymph node metastases and predict poor outcome in breast carcinoma. Clinical Cancer Research, 11(8), 2969-2973.

70. Calvo, F., et al. (1987). Human breast cancer cells share antigens with the myeloid monocyte lineage. British Journal of Cancer, 56(1), 15-19.

71. Shabo, I., et al. (2008). Breast cancer expression of CD163, a macrophage scavenger receptor, is related to early distant recurrence and reduced patient survival. International Journal of Cancer, 123(4), 780-786.

72. Heidemann, J., et al. (2002). Signet-ring cell carcinoma of unknown primary location. Metastatic to lower back musculature - remission following FU/FA chemotherapy. Zeitschrift für Gastroenterologie, 40(1), 33-36.

73. Hedley, D. W., Leary, J. A., \& Kirsten, F. (1985). Metastatic adenocarcinoma of unknown primary site: Abnormalities of 
cellular DNA content and survival. European Journal of Cancer \& Clinical Oncology, 21(2), 185-189.

74. Chandrasoma, P. (1980). Polymorph phagocytosis by cancer cells in an endometrial adenoacanthoma. Cancer, 45(9), 23482351.

75. Caruso, R. A., et al. (2002). Morphological evidence of neutrophil-tumor cell phagocytosis (cannibalism) in human gastric adenocarcinomas. Ultrastructural Pathology, 26(5), 315-321.

76. Ji, Y., et al. (1999). Effect of cell fusion on metastatic ability of mouse hepatocarcinoma cell lines. World Journal of Gastroenterology, 5(1), 22-24.

77. DeSimone, P. A., East, R., \& Powell, R. D., Jr. (1980). Phagocytic tumor cell activity in oat cell carcinoma of the lung. Human Pathology, 11(5 Suppl), 535-539.

78. Falini, B., et al. (1980). Erythrophagocytosis by undifferentiated lung carcinoma cells. Cancer, 46(5), 1140-1145.

79. Molad, Y., et al. (1991). Hemophagocytosis by small cell lung carcinoma. American Journal of Hematology, 36(2), 154-156.

80. Richters, A., Sherwin, R. P., \& Richters, V. (1971). The lymphocyte and human lung cancers. Cancer Research, 31(3), 214-222.

81. Ruff, M. R., \& Pert, C. B. (1984). Small cell carcinoma of the lung: Macrophage-specific antigens suggest hemopoietic stem cell origin. Science, 225(4666), 1034-1036.

82. Gazdar, A. F., et al. (1985). Origin of human small cell lung cancer. Science, 229(4714), 679-680.

83. Ruff, M. R., \& Pert, C. B. (1985). Origin of human small cell lung cancer. Science, 229(4714), 680.

84. Bunn, P. A., Jr., et al. (1985). Small cell lung cancer, endocrine cells of the fetal bronchus, and other neuroendocrine cells express the Leu-7 antigenic determinant present on natural killer cells. Blood, 65(3), 764-768.

85. Koren, H. S., Handwerger, B. S., \& Wunderlich, J. R. (1975). Identification of macrophage-like characteristics in a cultured murine tumor line. Journal of Immunology, 114(2 pt 2), 894-897.

86. Amaravadi, R. K., et al. (2007). Autophagy inhibition enhances therapy-induced apoptosis in a Myc-induced model of lymphoma. The Journal of Clinical Investigation, 117(2), 326-336.

87. Radosevic, K., et al. (1995). Occurrence and a possible mechanism of penetration of natural killer cells into K562 target cells during the cytotoxic interaction. Cytometry, 20(4), 273280.

88. Kerbel, R. S., et al. (1983). Spontaneous fusion in vivo between normal host and tumor cells: Possible contribution to tumor progression and metastasis studied with a lectin-resistant mutant tumor. Molecular and Cellular Biology, 3(4), 523-538.

89. Larizza, L., Schirrmacher, V., \& Pfluger, E. (1984). Acquisition of high metastatic capacity after in vitro fusion of a nonmetastatic tumor line with a bone marrow-derived macrophage. The Journal of Experimental Medicine, 160(5), 1579-1584.

90. De Baetselier, P., et al. (1984). Nonmetastatic tumor cells acquire metastatic properties following somatic hybridization with normal cells. Cancer and Metastasis Reviews, 3(1), 5-24.

91. De Baetselier, P., et al. (1984). Generation of invasive and metastatic variants of a non-metastatic T-cell lymphoma by in vivo fusion with normal host cells. International Journal of Cancer, 34(5), 731-738.

92. Lugini, L., et al. (2003). Potent phagocytic activity discriminates metastatic and primary human malignant melanomas: A key role of ezrin. Laboratory Investigation, 83(11), 1555-1567.

93. Lugini, L., et al. (2006). Cannibalism of live lymphocytes by human metastatic but not primary melanoma cells. Cancer Research, 66(7), 3629-3638.

94. Fais, S. (2004). A role for ezrin in a neglected metastatic tumor function. Trends in Molecular Medicine, 10(6), 249-250.
95. Breier, F., et al. (1999). Primary invasive signet-ring cell melanoma. Journal of Cutaneous Pathology, 26(10), 533-536.

96. Monteagudo, C., et al. (1997). Erythrophagocytic tumour cells in melanoma and squamous cell carcinoma of the skin. Histopathology, 31(4), 367-373.

97. Chakraborty, A. K., et al. (2000). A spontaneous murine melanoma lung metastasis comprised of host $\times$ tumor hybrids. Cancer Research, 60(9), 2512-2519.

98. Chakraborty, A. K., et al. (2001). Human monocyte $\times$ mouse melanoma fusion hybrids express human gene. Gene, 275(1), 103-106.

99. Brocker, E. B., Suter, L., \& Sorg, C. (1984). HLA-DR antigen expression in primary melanomas of the skin. The Journal of Investigative Dermatology, 82(3), 244-247.

100. Facchetti, F., Bertalot, G., \& Grigolato, P. G. (1991). KP1 (CD 68) staining of malignant melanomas. Histopathology, 19(2), 141-145.

101. Munzarova, M., Rejthar, A., \& Mechl, Z. (1991). Do some malignant melanoma cells share antigens with the myeloid monocyte lineage? Neoplasma, 38(4), 401-405.

102. Busund, L. T., et al. (2003). Spontaneously formed tumorigenic hybrids of Meth A sarcoma cells and macrophages in vivo. International Journal of Cancer, 106(2), 153-159.

103. Savage, D. G., et al. (2004). Hemophagocytic, non-secretory multiple myeloma. Leukaemia \& Lymphoma, 45(5), 1061-1064.

104. Andersen, T. L., et al. (2010). Myeloma cell-induced disruption of bone remodelling compartments leads to osteolytic lesions and generation of osteoclast-myeloma hybrid cells. British Journal of Haematology, 148(4), 551-561.

105. Yasunaga, M., et al. (2008). Ovarian undifferentiated carcinoma resembling giant cell carcinoma of the lung. Pathology International, 58(4), 244-248.

106. Talmadge, J. E., Key, M. E., \& Hart, I. R. (1981). Characterization of a murine ovarian reticulum cell sarcoma of histiocytic origin. Cancer Research, 41(4), 1271-1280.

107. Khayyata, S., Basturk, O., \& Adsay, N. V. (2005). Invasive micropapillary carcinomas of the ampullo-pancreatobiliary region and their association with tumor-infiltrating neutrophils. Modern Pathology, 18(11), 1504-1511.

108. Schorlemmer, H. U., et al. (1988). Similarities in function between pancreatic tumor cells and macrophages and their inhibition by murine monoclonal antibodies. Behring Institute Mitteilungen, 82, 240-264.

109. Imai, S., et al. (1981). Giant cell carcinoma of the pancreas. Acta Pathologica Japonica, 31(1), 129-133.

110. Shabo, I., et al. (2009). Expression of the macrophage antigen CD163 in rectal cancer cells is associated with early local recurrence and reduced survival time. International Journal of Cancer, 125(8), 1826-1831.

111. Chetty, R., \& Cvijan, D. (1997). Giant (bizarre) cell variant of renal carcinoma. Histopathology, 30(6), 585-587.

112. Chakraborty, A., et al. (2004). Donor DNA in a renal cell carcinoma metastasis from a bone marrow transplant recipient. Bone Marrow Transplantation, 34(2), 183-186.

113. Yilmaz, Y., et al. (2005). Donor Y chromosome in renal carcinoma cells of a female BMT recipient: Visualization of putative BMT-tumor hybrids by FISH. Bone Marrow Transplantation, 35(10), 1021-1024.

114. Etcubanas, E., et al. (1989). Rhabdomyosarcoma, presenting as disseminated malignancy from an unknown primary site: A retrospective study of ten pediatric cases. Medical and Pediatric Oncology, 17(1), 39-44.

115. Tsoi, W. C., \& Feng, C. S. (1997). Hemophagocytosis by rhabdomyosarcoma cells in bone marrow. American Journal of Hematology, 54(4), 340-342. 
116. Fais, S. (2007). Cannibalism: A way to feed on metastatic tumors. Cancer Letters, 258(2), 155-164.

117. Matarrese, P., et al. (2008). Xeno-cannibalism as an exacerbation of self-cannibalism: A possible fruitful survival strategy for cancer cells. Current Pharmaceutical Design, 14(3), 245-252.

118. Overholtzer, M., \& Brugge, J. S. (2008). The cell biology of cellin-cell structures. Nature Reviews Molecular Cell Biology, 9(10), 796-809.

119. Gupta, K., \& Dey, P. (2003). Cell cannibalism: Diagnostic marker of malignancy. Diagnostic Cytopathology, 28(2), 8687.

120. Duelli, D., \& Lazebnik, Y. (2003). Cell fusion: A hidden enemy? Cancer Cell, 3(5), 445-448.

121. Warner, T. F. (1975). Cell hybridizaiton: An explanation for the phenotypic diversity of certain tumours. Medical Hypotheses, 1 (1), 51-57.

122. Munzarova, M., Lauerova, L., \& Capkova, J. (1992). Are advanced malignant melanoma cells hybrids between melanocytes and macrophages? Melanoma Research, 2(2), 127-129.

123. Lu, X., \& Kang, Y. (2009). Cell fusion as a hidden force in tumor progression. Cancer Research, 69(22), 8536-8539.

124. Duelli, D., \& Lazebnik, Y. (2007). Cell-to-cell fusion as a link between viruses and cancer. Nature Reviews Cancer, 7(12), 968976.

125. Pawelek, J. M. (2005). Tumour-cell fusion as a source of myeloid traits in cancer. The Lancet Oncology, 6(12), 988-993.

126. Chettibi, S., \& Ferguson, M. (1999). Wound repair: An overview. In J. Snyderman (Ed.), Inflammation: Basic principles and clinical correlates (pp. 865-81). New York: Lippincott Williams \& Wilkins.

127. Sunderkotter, C., et al. (1994). Macrophages and angiogenesis. Journal of Leukocyte Biology, 55(3), 410-422.

128. Martin, P., \& Leibovich, S. J. (2005). Inflammatory cells during wound repair: The good, the bad and the ugly. Trends in Cell Biology, 15(11), 599-607.

129. Vignery, A. (2000). Osteoclasts and giant cells: Macrophagemacrophage fusion mechanism. International Journal of Experimental Pathology, 81(5), 291-304.

130. Bellingan, G. J., et al. (1996). In vivo fate of the inflammatory macrophage during the resolution of inflammation: Inflammatory macrophages do not die locally, but emigrate to the draining lymph nodes. Journal of Immunology, 157(6), 2577-2585.

131. Serhan, C. N., \& Savill, J. (2005). Resolution of inflammation: The beginning programs the end. Nature Immunology, 6(12), 1191-1197.

132. Diment, S., Leech, M. S., \& Stahl, P. D. (1988). Cathepsin D is membrane-associated in macrophage endosomes. The Journal of Biological Chemistry, 263(14), 6901-6907.

133. Stehle, G., et al. (1997). Plasma protein (albumin) catabolism by the tumor itself-Implications for tumor metabolism and the genesis of cachexia. Critical Reviews in Oncology/Hematology, 26(2), 77-100

134. Steinhaus, J. (1981). Ueber carcinom-einschlusse. Virchows Archiv, 126, 533-535.

135. Mizushima, N., et al. (2008). Autophagy fights disease through cellular self-digestion. Nature, 451(7182), 1069-1075.

136. Klionsky, D. J. (2004). Cell biology: Regulated self-cannibalism. Nature, 431(7004), 31-32.

137. Gotway, M.B., Conomos, P.J.,Bremner, R.M..Pleural metastatic disease from glioblastoma multiforme. Journal of Thoracic Imaging (in press).

138. Rubinstein, L. J. (1972). Tumors of the central nervous system. Washington: Armed Forces Institute of Pathology. 400.

139. Laerum, O. D., et al. (1984). Invasiveness of primary brain tumors. Cancer and Metastasis Reviews, 3(3), 223-236.
140. Taha, M., et al. (2005). Extra-cranial metastasis of glioblastoma multiforme presenting as acute parotitis. British Journal of Neurosurgery, 19(4), 348-351.

141. Hoffman, H. J., \& Duffner, P. K. (1985). Extraneural metastases of central nervous system tumors. Cancer, 56(7 Suppl), 1778-1782.

142. Ng, W. H., Yeo, T. T., \& Kaye, A. H. (2005). Spinal and extracranial metastatic dissemination of malignant glioma. Journal of Clinical Neuroscience, 12(4), 379-382.

143. Ghoneum, M., et al. (2005). Human squamous cell carcinoma of the tongue and colon undergoes apoptosis upon phagocytosis of Saccharomyces cerevisiae, the baker's yeast, in vitro. Anticancer Research, 25(2A), 981-989.

144. Mukherjee, P., Abate, L. E., \& Seyfried, T. N. (2004). Antiangiogenic and proapoptotic effects of dietary restriction on experimental mouse and human brain tumors. Clinical Cancer Research, 10(16), 5622-5629.

145. Mukherjee, P., et al. (2002). Dietary restriction reduces angiogenesis and growth in an orthotopic mouse brain tumour model. British Journal of Cancer, 86(10), 1615-1621.

146. Seyfried, T. N., \& Mukherjee, P. (2005). Anti-angiogenic and pro-apoptotic effects of dietary restriction in experimental brain cancer: Role of glucose and ketone bodies. In G. G. Meadows (Ed.), Integration/Interaction of oncologic growth. New York: Kluwer Academic.

147. Zhou, W., et al. (2007). The calorically restricted ketogenic diet, an effective alternative therapy for malignant brain cancer. Nutrition and Metabolism (London), 4, 5.

148. Marsh, J., Mukherjee, P., \& Seyfried, T. N. (2008). Aktdependent proapoptotic effects of caloric restriction on latestage management of a PTEN/TSC2-deficient mouse astrocytoma. Proceedings of the American Association for Cancer Research, 99, 1250.

149. Dong, W., et al. (1998). Altered alveolar macrophage function in calorie-restricted rats. American Journal of Respiratory Cell and Molecular Biology, 19(3), 462-469.

150. Zimmer, C., et al. (1995). MR imaging of phagocytosis in experimental gliomas. Radiology, 197(2), 533-538.

151. Camargo, F. D., Chambers, S. M., \& Goodell, M. A. (2004). Stem cell plasticity: From transdifferentiation to macrophage fusion. Cell Proliferation, 37(1), 55-65.

152. Camargo, F. D., Finegold, M., \& Goodell, M. A. (2004). Hematopoietic myelomonocytic cells are the major source of hepatocyte fusion partners. The Journal of Clinical Investigation, 113(9), 1266-1270

153. Parris, G. E. (2005). The role of viruses in cell fusion and its importance to evolution, invasion and metastasis of cancer clones. Medical Hypotheses, 64(5), 1011-1014.

154. Mekler, L. B. (1971). Hybridization of transformed cells with lymphocytes as 1 of the probable causes of the progression leading to the development of metastatic malignant cells. Vestnik Akademii Meditsinskikh Nauk SSSR, 26(8), 80-89.

155. Rachkovsky, M., \& Pawelek, J. (1999). Acquired melanocyte stimulating hormone-inducible chemotaxis following macrophage fusion with Cloudman S91 melanoma cells. Cell Growth \& Differentiation, 10(7), 517-524.

156. Ades, L., Guardiola, P., \& Socie, G. (2002). Second malignancies after allogeneic hematopoietic stem cell transplantation: New insight and current problems. Blood Reviews, 16(2), 135146.

157. Guillemin, G. J., \& Brew, B. J. (2004). Microglia, macrophages, perivascular macrophages, and pericytes: A review of function and identification. Journal of Leukocyte Biology, 75(3), 388-397.

158. Seyfried, T.N., Shelton, L.M., Mukherjee, P. (2010) Does the existing standard of care increase glioblastoma energy metabolism? Lancet Oncology, 11(9), 811-813. 
159. Pavlidis, N., \& Fizazi, K. (2009). Carcinoma of unknown primary (CUP). Critical Reviews in Oncology/Hematology, 69 (3), 271-278.

160. Carlson, H. R. (2009). Carcinoma of unknown primary: Searching for the origin of metastases. Jaapa, 22(8), 18-21.

161. Cuezva, J. M., et al. (2002). The bioenergetic signature of cancer: A marker of tumor progression. Cancer Research, 62 (22), 6674-6681.

162. Galluzzi, L., et al. (2010). Mitochondrial gateways to cancer. Molecular Aspects of Medicine, 31(1), 1-20.

163. John, A. P. (2001). Dysfunctional mitochondria, not oxygen insufficiency, cause cancer cells to produce inordinate amounts of lactic acid: The impact of this on the treatment of cancer. Medical Hypotheses, 57(4), 429-431.

164. Ramanathan, A., Wang, C., \& Schreiber, S. L. (2005). Perturbational profiling of a cell-line model of tumorigenesis by using metabolic measurements. Proceedings of the National Academy of Sciences of the United States of America, 102(17), 5992-5997.

165. Chen, Y., et al. (2009). Oxygen consumption can regulate the growth of tumors, a new perspective on the Warburg effect. PLoS ONE, 4(9), e7033.

166. Seyfried, T. N., \& Mukherjee, P. (2005). Targeting energy metabolism in brain cancer: Review and hypothesis. Nutrition and Metabolism (London), 2, 30.

167. Butow, R. A., \& Avadhani, N. G. (2004). Mitochondrial signaling: The retrograde response. Molecular Cell, 14(1), 115.

168. Singh, K. K., et al. (2005). Inter-genomic cross talk between mitochondria and the nucleus plays an important role in tumorigenesis. Gene, 354, 140-146.

169. Soto, A. M., \& Sonnenschein, C. (2004). The somatic mutation theory of cancer: Growing problems with the paradigm? Bioessays, 26(10), 1097-1107.

170. Lewis, C., \& Murdoch, C. (2005). Macrophage responses to hypoxia: Implications for tumor progression and anti-cancer therapies. The American Journal of Pathology, 167(3), 627-635.

171. DeBerardinis, R. J., et al. (2007). Beyond aerobic glycolysis: Transformed cells can engage in glutamine metabolism that exceeds the requirement for protein and nucleotide synthesis. Proceedings of the National Academy of Sciences of the United States of America, 104(49), 19345-19350.
172. Newsholme, P. (2001). Why is L-glutamine metabolism important to cells of the immune system in health, postinjury, surgery or infection? The Journal of Nutrition, 131(9 Suppl), 2515S2522S. discussion 2523S-4S.

173. Detmer, S. A., \& Chan, D. C. (2007). Functions and dysfunctions of mitochondrial dynamics. Nature Reviews Molecular Cell Biology, 8(11), 870-879.

174. Nygren, J. M., et al. (2008). Myeloid and lymphoid contribution to non-haematopoietic lineages through irradiation-induced heterotypic cell fusion. Nature Cell Biology, 10(5), 584-592.

175. Johansson, C. B., et al. (2008). Extensive fusion of haematopoietic cells with Purkinje neurons in response to chronic inflammation. Nature Cell Biology, 10(5), 575-583.

176. Chen, E. H., et al. (2007). Cell-cell fusion. FEBS Lett, 581, 2181-2193.

177. Dvorak, H. F. (1986). Tumors: Wounds that do not heal. Similarities between tumor stroma generation and wound healing. The New England Journal of Medicine, 315(26), 1650-1659.

178. D'Agostino, D. M., et al. (2005). Mitochondria as functional targets of proteins coded by human tumor viruses. Advances in Cancer Research, 94, 87-142.

179. Clippinger, A. J., \& Bouchard, M. J. (2008). Hepatitis B virus HBx protein localizes to mitochondria in primary rat hepatocytes and modulates mitochondrial membrane potential. Journal of Virology, 82(14), 6798-6811.

180. Koike, K. (2009). Hepatitis B virus X gene is implicated in liver carcinogenesis. Cancer Letters, 286(1), 60-68.

181. Smith, A. E., \& Kenyon, D. H. (1973). A unifying concept of carcinogenesis and its therapeutic implications. Oncology, 27(5), 459-479.

182. Glinsky, G. V., Berezovska, O., \& Glinskii, A. B. (2005). Microarray analysis identifies a death-from-cancer signature predicting therapy failure in patients with multiple types of cancer. The Journal of Clinical Investigation, 115(6), 1503-1521.

183. Willenbring, H., et al. (2004). Myelomonocytic cells are sufficient for therapeutic cell fusion in liver. Natural Medicines, 10(7), 744-748.

184. Harris, H. (1988). The analysis of malignancy by cell fusion: The position in 1988. Cancer Research, 48(12), 3302-3306.

185. Shelton, L. M., et al. (2010). A novel pre-clinical in vivo mouse model for malignant brain tumor growth and invasion. Journal Neurooncol, 99, 165-176. 